\title{
PASSIVE SAMPLING OF PHARMACEUTICALS AND PERSONAL CARE PRODUCTS IN AQUATIC ENVIRONMENTS
}

\author{
ZDENA KŘESINOVÁ1,2, KLÁRA PETR ${ }^{1,2}$, OND ŘEJ LHOTSKÝ1,3, \\ TORGEIR RODSAND ${ }^{4}$, and TOMÁŠ CAJTHAML ${ }^{1,2, *}$
}

\author{
${ }^{1}$ Institute for Environmental Studies, Faculty of Science, Charles University of Prague, Benátská 2, CZ-128 01 Prague 2, Czech Republic \\ 2 Institute of Microbiology Academy of Sciences of the Czech Republic, Vídeňská 1083, CZ-142 20 Prague 4, Czech Republic \\ ${ }^{3}$ DEKONTA a.s., Volutová 2523, CZ-158 00 Prague 5, Czech Republic \\ ${ }^{4}$ ALS Laboratory Group Norway AS, Drammensveien 173, N-0214 Oslo, Norway \\ *Corresponding author: cajthaml@biomed.cas.cz
}

\section{ABSTRACT}

Passive sampling is a rapidly developing technology, which is widely used for the monitoring of pollutants in different environments. Passive sampling offers significant advantages over traditional grab sampling. In the present review, the authors summarize the current literature on the methods of passive sampling used in the environmental monitoring of polar or semi-polar compounds in aqueous matrices. Methods of calibrating, design and deployment of samplers are also discussed. A major focus of this review is the use of polar organic compound integrative samplers (POCIS) and their use in sampling and monitoring of pharmaceuticals and personal care products (PCPs) in both equilibrium and non-equilibrium conditions.

Keywords: passive sampling, polar organic chemical integrative samplers, aquatic matrices, pharmaceuticals, personal care products

doi: $10.14712 / 23361964.2016 .8$

\section{Introduction}

Since its invention more than four decades ago, passive sampling has been widely used for the purpose of environmental monitoring in different media (e.g. soil, air, sediments and water). Despite its relatively long history (first use of passive sampling is reported in 1980s (Palmes and Gunnison 1973; Fowler 1982; Rose and Perkins 1982)), passive sampling is still developing and there are numerous review articles on this topic. Historically, passive sampling based on the principle of diffusion dosimeters was used for monitoring toxic chemicals in workplaces (Palmes and Gunnison 1973). Pine needles are one of the first "passive sampling devices", whose analysis is a well-established method for monitoring organic chemicals in the air (Kylin et al. 1994). Some organisms may also serve as "passive samplers" in the aquatic environment. They are sometimes called biological "dosimeters" and can be used as an indicator of the level of contamination in the aquatic biosphere (Schilderman et al. 1999).

The monitoring of trace levels of organic contaminants in water bodies is an ongoing challenge and has become possible only recently due to significant improvements in analytical techniques. Many of the environmental contaminants, often called 'emerging contaminants', are polar or semi-polar compounds such as pharmaceuticals and personal care products (PCPs). Thus, the behaviour and fate of these pollutants in the environment can be very different from the previously studied persistent organic pollutants (e.g. polychlorinated biphenyl or polyaromatic hydrocarbons). Contaminant concentrations can vary substantially because of the spatial and temporal variability of the source, pathways of its sorption and desorption and the pattern of its degradation and dissolution. Also, biogeochemical processes and human activities can influence the concentration of pollutants at point sources. Generally, the assessment of pollution is based on concentrations determined by analytical methods and/or toxicity data and biological methods. This means that in order to assess the quality of the environment, a large number of samples has to be analyzed to determine daily, monthly and/or annual time-weighted average concentrations (TWA) of the pollutants of interest. This monitoring approach can be prohibitively expensive and, depending on the technique used, can also very often be unfriendly to the environment.

Passive sampling, as a low-tech and cost-effective technique, represents a promising monitoring tool, which could avoid almost every disadvantage of active sampling and/or of the methods of preparing the samples. As an analytical tool, passive sampling is used to achieve some of the most basic steps in preparing samples. These could include pre-concentrating the analytes in order to increase the detection limits during measurements, reducing or eliminating solvent consumption (green chemistry) and elimination or reduction of matrix interferences. In most cases, passive sampling greatly simplify sample collection and preparation by eliminating training for device handling, need for power sources for their operation, provide a significant reduction in the cost of analysis and protection of analytes during transport and storage. Furthermore, many passive samplers can easily 
provide TWA concentrations, which is hardly achievable using active methods of sampling.

The first passive sampling of liquid media was used to monitor dissolved inorganic compounds in the surface water in an enclosed dialysis membrane (Benes and Steinnes 1974). First use of semi-permeable membrane devices (SPMDs) for sampling organic compounds was reported in 1990s (Huckins et al. 1990). Since then, many passive sampling devices have been developed and many of them are currently available commercially. Designing of passive samplers and their application in environmental analysis are described in several reviews (Vrana et al. 2005; Kot-Wasik et al. 2007; Seethapathy et al. 2008; Zabiegala et al. 2010). However, there are still very few publications on the use of passive sampling for determining pharmaceuticals and personal care products (see Fig. 1).

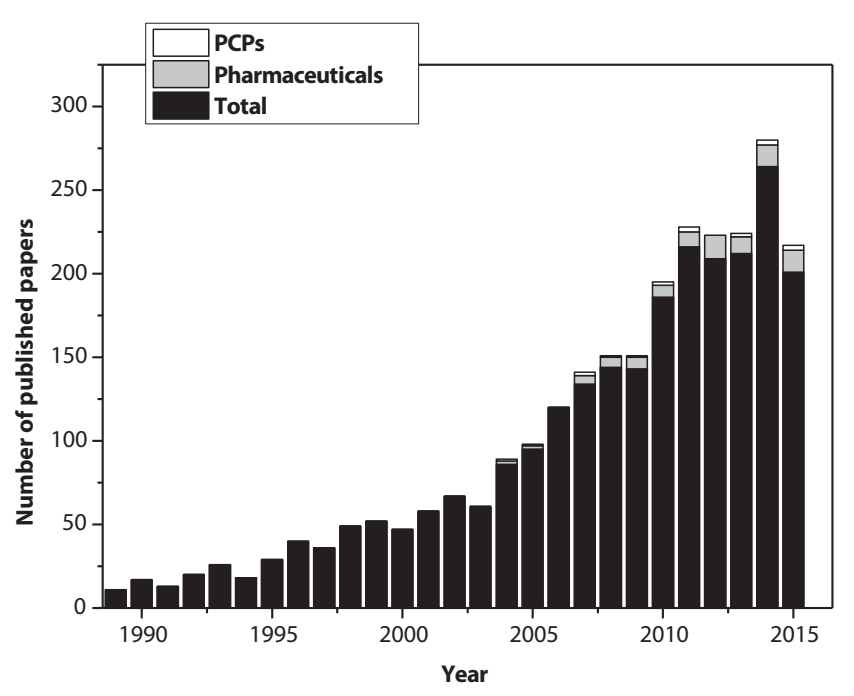

Fig. 1 Number of articles published on the application of passive sampling in aquatic environments between 1989 and 2015 with the number that focused on personal care products (PCPs) and pharmaceuticals indicated at the top of the figure.

In passive sampling, there are numerous variables that need to be considered (e.g. form of the analyte(s) of interest, duration of sampling, environmental parameters, chemical and physico-chemical properties of the analyte(s), medium or matrix to be sampled, type of measurement, whether quantitative or semi quantitative, cost and availability etc.). Moreover, there are often situations when a combination of passive samplers need to be deployed in order to obtain the relevant data. The physical deployment of passive samplers is simple but the sampling strategy involved can be more complicated and should therefore include correct assessment of the type of passive sampler or combination thereof; the exact location, time and durations of exposure and the analytical assessment. Knowledge of the type of pollution, its source, its potential fate in the environment together with the deployed analytical methods and their limita- tions are also important factors that need to be considered for a proper interpretation of data.

Passive sampling mechanisms are fairly well described for non-polar compounds (e.g. polychlorinated biphenyls, polyaromatic hydrocarbons, pesticides) but the mechanisms involved in sampling polar compounds are not fully characterized in terms of modelling of the uptake rates and various other effects of environmental factors.

\section{Passive Sampling}

\section{Types of Passive Samplers}

Passive sampling can be defined as any sampling technique based on the free flow of analytes from the sampled medium to a receiving phase in a sampling device, which results from a difference between the chemical potentials of the analyte in the two media under consideration (e.g. water and sorbent). The type of information obtained from passive sampling depends, to a large extent, on the regimes in which passive samplers operate during exposure in the field. There are two types of passive sampling devices, samplers in which target analytes dissolve (e.g. absorption) and those in which analytes are adsorbed (e.g. surface bonding); but the sampling process is very similar in both types of sampler. Once they are exposed to water, accumulation of analytes in the receiving phase occur by diffusion through a static layer of water in well-defined openings in the case of diffusion samplers, or by permeation through a porous or non-porous membrane in the case of permeation samplers.

1. The first type of sampler is also referred to as a partition sampler because it is based on partition theory. These samplers can achieve equilibrium between the sampler and the media if they are exposed for long enough. The material used in the partition passive sampler is selected in such a way that the test compounds dissolve in it much better than in water and therefore become highly concentrated and, as a result, are easier to measure. Partition samplers are often called hydrophobic samplers because they are generally used for non-polar compounds. Thus, these devices rely on the diffusion of the compounds to reach equilibrium between the sampler and the water.

2. The second type of sampler is known as an adsorption sampler. In this case, compounds bond very strongly to the adsorption material present in the sampler. Sorption capacity of the material in the sampler is usually very high, thus no equilibrium is reached. Samplers often bond polar compounds very strongly and are therefore frequently referred to as polar samplers or kinetic/sink samplers. Thus, these devices rely on diffusion and sorption to accumulate compounds in the sampler and remove and accumulate the compounds from water during the deployment period. 
The transport of target analytes from water to both types of passive sampler is diffusion-controlled, so that only freely dissolved substances are taken up or adsorbed and the variables in the uptake process for partition samplers are well known. The amount taken up by the partition sampler can therefore be used to calculate the concentration in the water phase. However, there are still a number of uncertain factors involved in the uptake process with regard to adsorption samplers and so there are also more uncertainties involved in the calculation of the concentration of analytes.

Whether a passive sampler is used in an equilibrium or non-equilibrium/kinetic mode also depends on:

- The exposure time of the passive samplers

- The concentration of target analytes

- The partitioning properties of target analytes

- The type of data that is to be obtained

\section{Principles of Passive Sampling}

Analytes in both types of samplers are trapped or retained in a suitable medium within the passive sampler, known as a reference or receiving phase. The receiving phase can be a solvent, chemical reagent or a porous adsorbent, which is exposed to the water phase during the sampling period. Dissolved analytes are not quantitatively extracted during the extraction process, but their adsorption or absorption generally follows the pattern shown in Fig. 2. The kinetics of exchange between a passive sampler and the water phase can be described by a first-order, one-compartment mathematical model:

$C_{s}(t)=C_{W} \frac{k_{1}}{k_{2}}\left(1-e^{-k_{2} t}\right)$,

where $C_{s}(\mathrm{t})$ is the concentration of the target analyte in the sampler at exposure time $t, C_{\mathrm{w}}$ is the concentration of

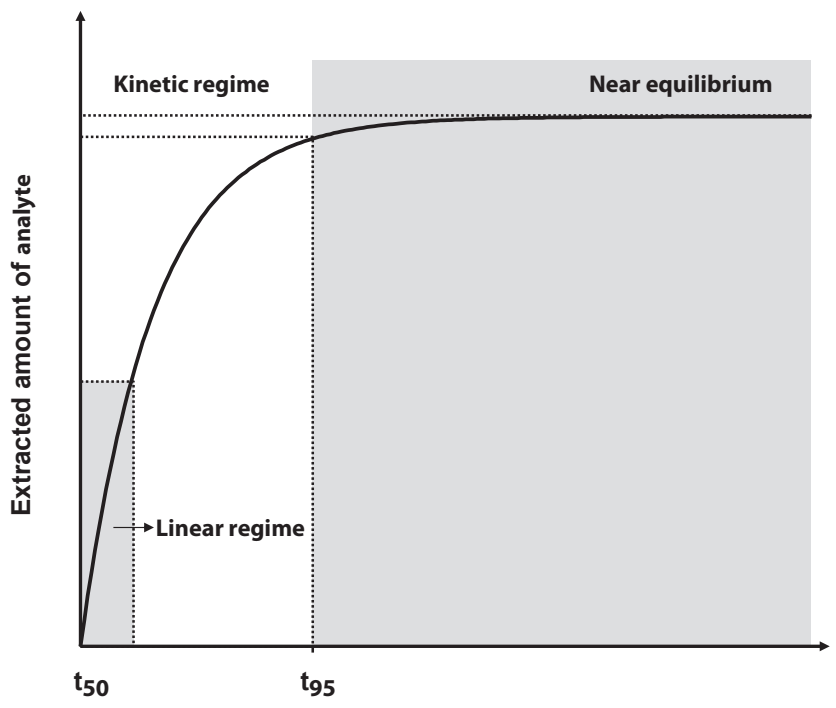

Fig. 2 Passive sampling devices operate in two mail regimes - kinetic and equilibrium. the target analyte in the aqueous environment (TWA the time-weighted average concentration of pollutant in the water phase), and $k_{1}$ and $k_{2}$ are the uptake and offload rate constants, respectively. Both main accumulation regimes, either kinetic or equilibrium, can be distinguished during the operation of the sampler in the field.

\section{Equilibrium Passive Samplers}

In equilibrium passive samplers, the exposure time is sufficiently long to establish thermodynamic equilibrium between the matrix (reference/receiving phase) and water. Equilibrium could be reached within seconds to months depending on the sampler, the compound and its concentration. The equation can be reduced to:

$C_{s}=C_{W} \frac{k_{1}}{k_{2}}=C_{W} K$

In this equation $K$ is the phase-water partition coefficient. The basic conditions of the equilibrium sampling technique is a known response time, after which a stable concentration is reached, and sampler capacity, which must be kept well below that of the sample to avoid depletion during extraction. The device response time should be shorter than any fluctuation in the sampled/sampling environment so that many environmental stressors do not effect the results.

The sampling times of different passive samplers range from seconds to months. The results obtained from equilibrium samplers are comparable with those obtained by grab sampling and therefore the device is not suitable for the determination of TWA concentrations (Kot-Wasik et al. 2007). Equilibrium passive samplers are often referred to as biological "dosimeters" (e.g. mussels, fish), because they mimic the part of the animal body where bioaccumulation and bioconcentration occurs. Samplers contain a receiving phase in which the contaminants are trapped as in living organisms. Equilibrium samplers provide the information about the level of contamination in the monitored environment instead of quantitative information on the concentration of pollutants.

Commercial products available:

- Regenerated-Cellulose Dialysis Membrane Samplers

- Nylon-Screen Passive Diffusion Samplers (NSPDS)

- Passive Vapor Diffusion Samplers (PVDs)

- Peeper Samplers

- Polyethylene Diffusion Bag Samplers (PDBs)

- Rigid Porous Polyethylene Samplers (RPPS)

- Diffusive Multi-layer Sampler (DMLS)

Passive samplers in equilibrium mode are used for monitoring DDT, $\mathrm{Hg}$ ions, chlorophenols, hexachlorobenzene, PCBs, anilines, pesticides, phenols, triclosan, PBDEs, biotoxins and PCDD/Fs (review in (Vrana et al. 2005; Kot-Wasik et al. 2007; Mills et al. 2007; Verreydt et al. 2010; Zabiegala et al. 2010; Lydy et al. 2014; Mills et al. 2014). 


\section{Kinetic Passive Samplers (Non-Equilibrium)}

Non-equilibrium/kinetic passive samplers do not reach equilibrium with the surrounding environment within the sampling period. Kinetic sampling permits the measurement of analyte concentrations over extended periods of time. In kinetic sampling, the rate of mass transfer to the receiving phase is linearly proportional to the difference between the chemical activity of the target analyte in the water phase and in the reference phase. The rate of desorption of target analyte from the receiving phase to water is negligible and during the period of exposure the initial rate of uptake of the sampler is linear. The equation can be reduced to:

$M_{s}(t)=C_{W} R_{S} t$,

where $M_{S}(t)$ is the mass of target analyte accumulated in the receiving phase after a certain exposure time $(t)$ and $R_{S}$ is the proportionality constant - sampling rate. $R_{S}$ may be interpreted as the volume of water cleared of analyte per unit exposure time by the device. $R_{S}$ is usually not affected by $C_{w}$ (the time-weighted average pollutant concentration in water phase), but can vary with water flow or turbulence, temperature and biofouling.

Kinetic samplers are characterized by high capacity for contaminants of interest. The high capacity ensures the continuous sampling throughout the exposure period. These samplers provide the TWA concentrations concentration of target analytes in the sample matrix averaged over a known period of time.

Commercially available samplers:

- Polar Organic Chemical Integrative Samplers (POCIS) for VOCs

- Semi-Permeable Membrane Devices (SPMDs) for lipophilic organic compounds

- GORE ${ }^{\mathrm{m}}$ Sorber Module

- Passive In-Situ Concentration Extraction Sampler (PISCES)

- Solid Phase Microextraction (SPME)

- Membrane-enclosed Sorptive Coating Sampler (MESCO)

Passive samplers in the kinetic mode have been recently used for monitoring DDT, endocrine disruptors, various pharmaceuticals, pesticides, personal care products, PAHs, PCBs, UV filters, plasticizers, organotin compounds etc. (review in Vrana et al. 2005; Kot-Wasik et al. 2007; Mills et al. 2007; Verreydt et al. 2010; Zabiegala et al. 2010; Harman et al. 2012; Amdany et al. 2014b; Lydy et al. 2014; Mills et al. 2014).

\section{Factors Affecting Passive Sampling}

The sampling rate is an important issue when linear (kinetic) uptake passive samplers are used for field sampling. Temperature, rate of water flow, salinity, $\mathrm{pH}$ and biofouling as well as the properties (e.g. $K_{\text {ow }}$ ) of some analytes are known to affect uptake. However the sampling rate could be a-priori determined in the laboratory dur- ing calibration or predicted by empirical equations and many parameters may have an important influence on it (Ouyang et al. 2007; Seethapathy et al. 2008).

Only analytes dissolved in the water can pass, by diffusion, the water boundary layer (WBL), which is generated due to the high viscosity at the surface of the sampler (Booij et al. 1998) and represents a rate-limiting step in the uptake into the receiving phase (except biofouling). The thickness of WBL is dependent on water flow or turbulence around the sampler and also depends on the type and properties of the membrane. These factors can significantly influence the rate of accumulation as is reported for non-polar samplers (Booij et al. 1998). After they cross the WBL analytes are transported across the membrane through the water-filled pores or via the polymer itself.

Finally, compounds are transferred to the sorbent material mainly via adsorption (polar substances) or absorption (non-polar substances). When water turbulence is high enough to make the resistance to mass transfer into the boundary layer negligible, transport through the membrane becomes the rate-limiting factor.

The influence of salinity and $\mathrm{pH}$ on $R_{S}$ is usually compound-specific and highly dependent on the various chemical groups present in the structure of the analyte. Increasing salinity increases the energy required for a solute's molecular cavity to form and increases the partitioning of neutral compounds toward non-aqueous phases. This is also called salting-out and it increases with the size and decreases with the polarity of the analyte (Endo et al. 2012). Similarly, the pH will affect the $R_{S}$ depending on the type of analyte, especially for basic analytes (Li et al. 2011). In several studies, very small effects of the water temperature is reported in the range of $5-25^{\circ} \mathrm{C}$ (twofold or less) (Li et al. 2011). Biofouling is the accumulation of microorganisms and various flora and fauna on wet surfaces, which may form a biofilm. For extended exposure (e.g. POCIS samplers), biofouling of the surface can influence the mass transfer by increasing the thickness of the barrier on the membrane and also by blocking the water-filled pores (Huckins et al. 2006). The thickness of the biofilm varies from exposure to exposure and also between spots on the same membrane and the effect, is thus, difficult to predict. The problem of biofouling of the membrane can be reduced by using suitable membrane materials. POCIS samplers made of polyethersulphone slowly bleed anti-fouling solvent (e.g. hexane) during exposure and as a consequence are less affected by biofouling (Alvarez et al. 2004).

\section{Calibration of Passive Samplers}

There are several different calibration methods for determining the sampling rate $\left(R_{S}\right)$ that vary in their level of complexity. Therefore, selecting the appropriate method depends on the research objective. Laboratory calibration is more often used than calibration in situ. However, 
calibration in the laboratory is a time-consuming procedure and it is impossible to encompass the wide range of environmental exposure conditions. Therefore a performance reference compound (PRC) is often used for calibration. The most often used calibration methods are:

- Static renewal - exposure of samplers to a small volume of fortified water, refreshed periodically to minimize the decrease in analyte concentration. The mass of the absorbed analyte is measured at intervals (Alvarez et al. 2004).

- Static depletion - exposure of samplers to single spiked (fortified) water and analysis of the analyte present in the water phase. Usually a high initial concentration is used and the depletion is monitored over several orders of magnitude (Bartelt-Hunt et al. 2011).

- Flow-through systems - exposure of several samplers in a tank filled with fortified water, which is continuously supplied. Samplers are removed from the tank over time and sorbents are analyzed in order to assess analyte uptake (Vrana et al. 2006).

- In situ calibration - measurement of sampling rate at the exact location where the sampling experiments are to be conducted. Deployed samplers are calibrated by comparing analyte accumulation with TWA concentration in water obtained from gram sampling (Harman et al. 2011).

- PRC based system - PRC are organic compounds that are introduced into the receiving phase of the sampler before it is exposed. PRC allows assessment of whether analytes are in equilibrium or in the kinetic phase and an estimate of $R_{\mathrm{S}}$ of the target analyte in situ as their dissipation shows isotropic kinetics analogous to analyte uptake (Booij et al. 1998).

\section{Biomagnification}

One of the major advantages of passive samplers used in equilibrium mode is that these devices can be used for determining biomagnification due to their similarities with biological systems (hydrophobic depots covered with a semi-permeable membrane). Thus the hydrophobic analytes are trapped in the receiving medium after the exposure of the device, as in living organisms. Sampling of indigenous or transplanted organisms (e.g. fish, microalgae, mussels) is an accepted practice in many programs focused on the monitoring of the aquatic environment (van der Oost et al. 2003). Although no passive sampler is a perfect model for biological organisms and concentration levels obtained from living organisms and passive samplers differ, SPMD samplers are often used to model biomagnification (Yargicoglu and Reddy 2015).

\section{POCIS Application}

The demand for the monitoring of polar substances in water in the past resulted in an increased interest among researches worldwide in elucidating the passive sampling processes occurring in POCIS. Latest reviews of Harman et al. and Morin et al. report on the performance of POCIS, in particular the calibration methods used from 1999 to 2012 (Harman et al. 2012; Morin et al. 2012). In the current review, the applications that focus on the passive sampling of pharmaceuticals and personal care products (PPCP) are from the middle of 2011 to 2015 (Table 1). The crucial issues of this sample processing, which is schematically demonstrated in Fig. 3, are discussed and the main features of the traditional POCIS design and the new prototypes are also described. Target analytes and the sampling sites of interests are considered and new findings on the method of calibration are highlighted. Analytical aspects of sample extraction, chemical analysis and biological testing are also included.

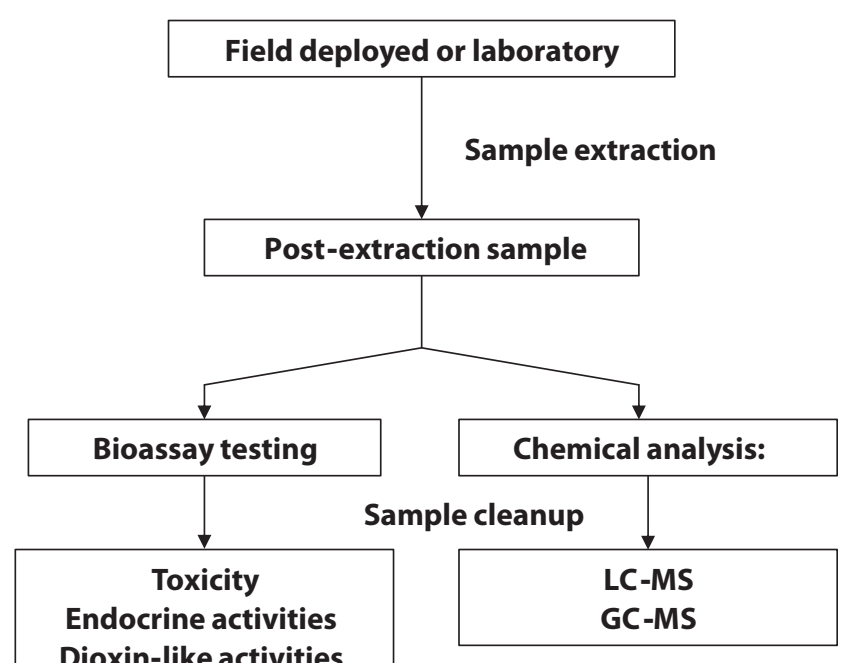

Fig. 3 POCIS processing scheme (modified according to Alvarez et al. 2008).

\section{POCIS Design}

The Polar Organic Chemical Integrative Samplers (POCIS) are designed to sample water-soluble organic chemicals from the aqueous environment. As mentioned above, this device relies on diffusion and adsorption to accumulate the analyte. The deployment time usually ranges between weeks and months.

POCIS typically consist of a sorbent, inserted between two microporous membranes that are assembled in a stainless steel housing (Fig. 4). Both sorbent and microporous membrane can vary depending on the particular application. Laboratory-derived calibration data (i.e., data regarding the sampling rate) are only applicable to devices having common surface area-to-sorbent mass ratios, resulting in the necessity of a standardized configuration.

Two different configurations of POCIS are commonly used, each containing a different sorbent. A "Pesticides" 
Table 1 Use of polar organic chemical integrative sampler for monitoring of PPCP from the middle of 2011 to 2015.

\begin{tabular}{|c|c|c|c|c|c|c|c|}
\hline $\begin{array}{l}\text { Aim of the } \\
\text { study }\end{array}$ & $\begin{array}{l}\text { Group of targeted } \\
\text { analytes }\end{array}$ & Matrix & $\begin{array}{l}\text { Analytical } \\
\text { technique }\end{array}$ & $\begin{array}{l}\text { Measured } \\
\text { concentrations / } \\
\text { maximal } \\
\text { exposure } \\
\text { duration (days) } \\
\end{array}$ & Sorbent & Note & Reference \\
\hline Screening & Antibiotics (vet.) & $\begin{array}{l}\text { Agricultural } \\
\text { watershed }\end{array}$ & LC-MS/MS & $\begin{array}{l}0.0003-68 \mathrm{ng} \mathrm{l}^{-1} \\
/ 52\end{array}$ & HLB & $\begin{array}{l}\text { Seasonal occurrence } \\
\text { screening }\end{array}$ & $\begin{array}{l}\text { (Jaimes-Correa } \\
\text { et al. 2015) }\end{array}$ \\
\hline Calibration & NSAIDs & $\begin{array}{l}\text { River water } \\
\text { WWTP effluent }\end{array}$ & LC-MS/MS & $\begin{array}{l}0.33-0.46 \mathrm{ng} \mathrm{l}^{-1} \\
/ 15\end{array}$ & HLB & $\begin{array}{l}\text { Stir bar sorptive } \\
\text { extraction develop- } \\
\text { ment } \\
\text { Laboratory calibra- } \\
\text { tion }\end{array}$ & $\begin{array}{l}\text { (Tanwar et al. } \\
\text { 2015) }\end{array}$ \\
\hline Screening & $\begin{array}{l}\text { NSAIDs, } \beta \text {-blockers, an- } \\
\text { tidepressants, anticon- } \\
\text { vulsives }\end{array}$ & WWTP effluent & LC-MS/MS & $\begin{array}{l}160-440 \text { ng sam- } \\
\operatorname{pler}^{-1}(\Sigma) / 31\end{array}$ & HLB & $\begin{array}{l}\text { Biological effect on } \\
\text { muscle tissue (PAH, } \\
\text { PCB) }\end{array}$ & $\begin{array}{l}\text { (Turja et al. } \\
\text { 2015) }\end{array}$ \\
\hline Screening & $\begin{array}{l}\text { Analgesics antidepres- } \\
\text { sants, calcium-channel } \\
\text { blockers, benzodiaze- } \\
\text { pines, NSAIDs, } \beta \text {-block- } \\
\text { ers, anti-histamines, } \\
\text { insecticides, antibacte } \\
\text { rials/antifungals, stimu- } \\
\text { lants, steroid hormones, } \\
\text { chemotherapeutics, } \\
\text { cannabinoids }\end{array}$ & WWTP effluent & $\begin{array}{l}\text { UHPLC-MS/ } \\
\text { MS }\end{array}$ & $\begin{array}{l}0.01 \pm 0.01-85.39 \\
\pm 4.98 \mathrm{ng} \mathrm{l}^{-1} / 30\end{array}$ & HLB & $\begin{array}{l}\text { Grab sampling in } \\
\text { parallel } \\
\text { Effects on fish }\end{array}$ & $\begin{array}{l}\text { (Zenobio et al. } \\
\text { 2015) }\end{array}$ \\
\hline Calibration & $\begin{array}{l}\text { NSAIDs } \\
\text { antibacterials/antifun- } \\
\text { gals }\end{array}$ & $\begin{array}{l}\text { WWTP influ- } \\
\text { ents } \\
\text { WWTP efluents }\end{array}$ & LC-UV(FLD) & $\begin{array}{l}52.3-127.7 \mu \mathrm{g} \mathrm{I}^{-1} \\
10.7-24.6 \mu \mathrm{g} \mathrm{I}^{-1} \\
/ 14\end{array}$ & HLB & $\begin{array}{l}\text { Uptake kinetics } \\
\text { Laboratory calibra- } \\
\text { tion }\end{array}$ & $\begin{array}{l}\text { (Amdany et al. } \\
\text { 2014a) }\end{array}$ \\
\hline Calibration & $\begin{array}{l}\text { Anticonvulsives, calci- } \\
\text { um-channel blockers, } \\
\text { anti-histamines, antip- } \\
\text { sychotics, chemother- } \\
\text { apeutics, fungicides, } \\
\text { anticonvulsives, steroid } \\
\text { hormones, hypolipidem- } \\
\text { ics, antibacterials }\end{array}$ & $\begin{array}{l}\text { Lake water } \\
\text { estuarine water }\end{array}$ & LC-MS/MS & $\begin{array}{l}0.0012-265 \mathrm{ng} \mathrm{l}^{-1} \\
/ 28\end{array}$ & HLB & $\begin{array}{l}\text { Tropical ecosystems } \\
\text { In situ calibration }\end{array}$ & $\begin{array}{l}\text { (Bayen et al. } \\
\text { 2014) }\end{array}$ \\
\hline Calibration & $\begin{array}{l}\text { Wide spectrum of phar- } \\
\text { maceuticals }\end{array}$ & $\begin{array}{l}\text { Spiked mineral } \\
\text { water }\end{array}$ & LC-MS & ND & HLB & $\begin{array}{l}\text { POCIS-Nylon } \\
\text { PRC, laboratory } \\
\text { calibration }\end{array}$ & $\begin{array}{l}\text { (Belles et al. } \\
\text { 2014) }\end{array}$ \\
\hline Forensic & Amphetamines & $\begin{array}{l}\text { Sewage } \\
\text { (sewer line) }\end{array}$ & LC-MS/MS & $>3 \mathrm{ng} \mathrm{ml}^{-1} / 27$ & HLB & $\begin{array}{l}\text { Forensic analysis } \\
\text { Proof of concept }\end{array}$ & $\begin{array}{l}\text { (Boles and } \\
\text { Wells, 2014) }\end{array}$ \\
\hline Screening & $\begin{array}{l}69 \text { compounds includ- } \\
\text { ing PCP }\end{array}$ & WWTP effluent & GC-MS & $5-500 \mathrm{ng} \mathrm{l}^{-1} / 29$ & HLB & $\begin{array}{l}\text { CLAM and discrete } \\
\text { sampler comparison }\end{array}$ & $\begin{array}{l}\text { (Coes et al. } \\
\text { 2014) }\end{array}$ \\
\hline $\begin{array}{l}\text { Screening } \\
\text { Bioassay }\end{array}$ & $\begin{array}{l}\text { Steroid hormones, anti- } \\
\text { biotics, NSAIDs }\end{array}$ & $\begin{array}{l}\text { Pharmaceutical } \\
\text { factory area } \\
\text { WWTP influent } \\
\text { WWTP effluent }\end{array}$ & LC & Up to $\mu \mathrm{g} \mathrm{I}^{-1} / 30$ & HLB & $\begin{array}{l}\text { Multireceptor bio- } \\
\text { assay-based mon- } \\
\text { itoring (hormone, } \\
\text { dioxin activity) }\end{array}$ & $\begin{array}{l}\text { (Creusot et al. } \\
\text { 2014) }\end{array}$ \\
\hline Calibration & NSAIDs & $\begin{array}{l}\text { River water } \\
\text { Drinking water }\end{array}$ & LC-MS/MS & $\begin{array}{l}0.11-0.67 \mathrm{ng} \mathrm{I}^{-1} \\
0.20-0.22 \mathrm{ng} \mathrm{l}^{-1} \\
/ 14\end{array}$ & HLB & $\begin{array}{l}\text { Recirculating flow } \\
\text { system for laborato- } \\
\text { ry calibration }\end{array}$ & $\begin{array}{l}\text { (Di Carro et al. } \\
\text { 2014) }\end{array}$ \\
\hline $\begin{array}{l}\text { Screening } \\
\text { Calibration }\end{array}$ & $\begin{array}{l}\text { Analgesics, psycholep- } \\
\text { tics, antidepressants, } \\
\text { illicit drugs }\end{array}$ & Surface water & LC-MS/MS & $\begin{array}{l}463 \text { to } 6447 \\
\text { ng sampler-1 / } 20\end{array}$ & $\begin{array}{l}\text { Tripha- } \\
\text { sic }\end{array}$ & $\begin{array}{l}\text { Czech aquatic envi- } \\
\text { ronment } \\
\text { In situ calibration }\end{array}$ & $\begin{array}{l}\text { (Fedorova et al. } \\
\text { 2014) }\end{array}$ \\
\hline Screening & $\begin{array}{l}\text { Anticonvulsives, } \\
\text { chemotherapeutics, } \\
\text { hypolipidemics, NSAIDs, } \\
\text { antibacterials, artificial } \\
\text { sweeteners }\end{array}$ & Sewage lagoon & LC-MS/MS & $\begin{array}{l}0.04 \pm 0.01-60.3 \pm \\
8.05 \mathrm{ng} \mathrm{l}^{-1} / 14\end{array}$ & HLB & $\begin{array}{l}\text { Seasonal changes in } \\
\text { removal } \\
\text { SPMD in parallel }\end{array}$ & $\begin{array}{l}\text { (Hoque et al. } \\
\text { 2014) }\end{array}$ \\
\hline Calibration & $\begin{array}{l}\text { Stimulants, anticonvul- } \\
\text { sives, antibacterials/ } \\
\text { antifungals, insecticides, } \\
\text { diuretics, analgesic }\end{array}$ & $\begin{array}{l}\text { Spiked potable } \\
\text { water }\end{array}$ & LC-MS & $\begin{array}{l}\text { LOQ 0.03-0.33 } \\
\mu \mathrm{g} \mathrm{I}^{-1} / 26\end{array}$ & Strata-X & $\begin{array}{l}\text { Chemcatchers and } \\
\text { grab samples in } \\
\text { parallel } \\
\text { Laboratory calibra- } \\
\text { tion }\end{array}$ & $\begin{array}{l}\text { (Kaserzon et al. } \\
\text { 2014) }\end{array}$ \\
\hline
\end{tabular}




\begin{tabular}{|c|c|c|c|c|c|c|c|}
\hline $\begin{array}{l}\text { Screening } \\
\text { Bioassay }\end{array}$ & $\begin{array}{l}\text { Wide spectrum of phar- } \\
\text { maceuticals }\end{array}$ & WWTP effluent & LC-MS & ND & $\begin{array}{l}\text { HLB } \\
\text { Tripha- } \\
\text { sic }\end{array}$ & $\begin{array}{l}\text { Anti-androgenic } \\
\text { activity; YAS } \\
\text { LDPE and silicone } \\
\text { strips in parallel }\end{array}$ & $\begin{array}{l}\text { (Liscio et al. } \\
\text { 2014) }\end{array}$ \\
\hline $\begin{array}{l}\text { Screening } \\
\text { Calibration }\end{array}$ & $\begin{array}{l}\text { Anticonvulsives, } \\
\text { chemotherapeutics, } \\
\text { NSAIDs, hypolipidemics, } \\
\text { steroid hormones and } \\
\text { sweeteners }\end{array}$ & $\begin{array}{l}\text { Drinking water } \\
\text { treatment } \\
\text { plants }\end{array}$ & LC-MS/MS & $\begin{array}{l}0.02 \pm 0.01-29.83 \\
\pm 6.03 \mathrm{ng} \mathrm{l}^{-1} / 30\end{array}$ & HLB & $\begin{array}{l}\text { Grab sampling in } \\
\text { parallel }\end{array}$ & $\begin{array}{l}\text { (Metcalfe et al. } \\
\text { 2014) }\end{array}$ \\
\hline Screening & $\begin{array}{l}\text { Amphetamines, cocaine } \\
\text { and its metabolite, } \\
\text { opioids }\end{array}$ & WWTP proces & LC-MS/MS & $\begin{array}{l}40 \pm 5-497 \pm 9 \\
\mathrm{ng} \mathrm{I}^{-1} \\
9 \pm 1-497 \pm 9 \\
\mathrm{ng} \mathrm{I}^{-1} / \mathrm{ND}\end{array}$ & HLB & $\begin{array}{l}\text { Removal efficiencies } \\
\text { Composite and grab } \\
\text { sampling in parallel }\end{array}$ & $\begin{array}{l}\text { (Rodayan et al. } \\
\text { 2014) }\end{array}$ \\
\hline $\begin{array}{l}\text { Screening } \\
\text { Calibration }\end{array}$ & $\begin{array}{l}\text { Sulfonamides, Antibiot- } \\
\text { ics, steroid hormones }\end{array}$ & Estuarine water & $\begin{array}{l}\text { UHPLC-MS/ } \\
\text { MS }\end{array}$ & $\begin{array}{l}\text { Below quantifica- } \\
\text { tion to } 1613 \mathrm{ng} \mathrm{I}^{-1} \\
\text { / } 60\end{array}$ & HLB & $\begin{array}{l}\text { In situ and laborato- } \\
\text { ry calibration } \\
\text { Salinity experiment }\end{array}$ & (Shi et al. 2014) \\
\hline $\begin{array}{l}\text { Screening } \\
\text { Calibration }\end{array}$ & $\begin{array}{l}\text { Amphetamines, cocaine } \\
\text { and its metabolite, } \\
\text { opioids }\end{array}$ & WWTP & LC-MS/MS & $\begin{array}{l}1 \pm 0.01-893 \pm \\
208 \mathrm{ng} \mathrm{l}^{-1} / 14\end{array}$ & ND & $\begin{array}{l}\text { Removal efficiencies } \\
\text { Composite sam- } \\
\text { pling in parallel }\end{array}$ & $\begin{array}{l}\text { (Yargeau et al. } \\
\text { 2014) }\end{array}$ \\
\hline $\begin{array}{l}\text { Screening } \\
\text { Calibration } \\
\text { Bioassay }\end{array}$ & Estrogens & Surface water & GC-MS/MS & $\begin{array}{l}\text { Mean } 1.9 \mathrm{ng} \mathrm{l}^{-1} \\
\text { (estrone) / } 44\end{array}$ & HLB & $\begin{array}{l}\text { Estrogenity assays } \\
\text { YES, T47D-KBluc } \\
\text { Assay, E-Screen } \\
\end{array}$ & $\begin{array}{l}\text { (Alvarez et al. } \\
\text { 2013) }\end{array}$ \\
\hline $\begin{array}{l}\text { Stability } \\
\text { study }\end{array}$ & $\begin{array}{l}\text { Wide range of pharma- } \\
\text { ceuticals and PCP }\end{array}$ & $\begin{array}{l}\text { Laboratory } \\
\text { samples, river } \\
\text { water }\end{array}$ & $\begin{array}{l}\text { UHPLC-MS/ } \\
\text { MS }\end{array}$ & $\begin{array}{l}6.9 \pm 1.3-2130 \pm \\
105 \text { ng sampler }^{-1} \\
\text { (storage } 255 \text { days) }^{2}\end{array}$ & HLB & $\begin{array}{l}\text { Stability on POCIS } \\
\text { and SPE cartridges }\end{array}$ & $\begin{array}{l}\text { (Carlson et al. } \\
\text { 2013) }\end{array}$ \\
\hline $\begin{array}{l}\text { Screening } \\
\text { Bioassay }\end{array}$ & $\begin{array}{l}\text { Endocrine disrupting } \\
\text { chemicals }\end{array}$ & Surface water & LC & - & HLB & $\begin{array}{l}\text { Endocrine and } \\
\text { dioxin-like activities } \\
\text { (ASE extracts) } \\
\text { MELN, MDA-kb2, } \\
\text { HG5LN-hPXR, PLHC- } \\
\text { 1, EROD } \\
\text { Sediment/water } \\
\text { distribution } \\
\text { SPMD in parallel }\end{array}$ & $\begin{array}{l}\text { (Creusot et al. } \\
\text { 2013) }\end{array}$ \\
\hline $\begin{array}{l}\text { Screening } \\
\text { Bioassay }\end{array}$ & $\begin{array}{l}\text { Sulfonamides, antibi- } \\
\text { otics, anticonvulsives, } \\
\text { NSAIDs }\end{array}$ & $\begin{array}{l}\text { WWTP in/ } \\
\text { effluent } \\
\text { River water up/ } \\
\text { downstream }\end{array}$ & LC-MS/MS & $\begin{array}{l}2-18,550 \text { ng sam- } \\
\text { pler-1 }(\Sigma)^{-1} \text { 3-272 ng sam- } \\
\operatorname{pler}^{-1}(\Sigma) / 23\end{array}$ & ND & $\begin{array}{l}\text { Estrogen-, andro- } \\
\text { gen- and aryl hy- } \\
\text { drocarbon receptor } \\
\text { mediated activities } \\
\text { H4IIE-luc, MVLN, } \\
\text { MDA-kb2 } \\
\text { Cytotoxicity } \\
\text { SPMD in parallel }\end{array}$ & $\begin{array}{l}\text { (Jalova et al. } \\
\text { 2013) }\end{array}$ \\
\hline $\begin{array}{l}\text { Screening } \\
\text { Calibration }\end{array}$ & $\begin{array}{l}\text { Chemotherapeutics, } \\
\text { NSAIDs, } \beta \text {-blockers, } \\
\text { corticosteroids }\end{array}$ & $\begin{array}{l}\text { Hospital } \\
\text { sewage }\end{array}$ & $\begin{array}{l}\text { UHPLC-MS/ } \\
\text { MS }\end{array}$ & 0.5 to $12 \mu \mathrm{g} \mathrm{I}^{-1} / 7$ & HLB & $\begin{array}{l}\text { Hospital wastewater } \\
\text { Laboratory calibra- } \\
\text { tion }\end{array}$ & $\begin{array}{l}\text { (Bailly et al. } \\
\text { 2013) }\end{array}$ \\
\hline Calibration & $\begin{array}{l}56 \text { compounds includ- } \\
\text { ing hormones and } \\
\text { pharmaceuticals }\end{array}$ & $\begin{array}{l}\text { Spiked tap } \\
\text { water }\end{array}$ & LC-MS/MS & - & HLB & $\begin{array}{l}\text { POCIS-derived accu- } \\
\text { mulation curves } \\
\text { Laboratory calibra- } \\
\text { tion }\end{array}$ & $\begin{array}{l}\text { (Morin et al. } \\
\text { 2013) }\end{array}$ \\
\hline Calibration & $\begin{array}{l}\text { Alkylphenols (sur- } \\
\text { factants), steroid } \\
\text { hormones }\end{array}$ & $\begin{array}{l}\text { Surface waters } \\
\text { WWTP influent } \\
\text { WWTP effluent }\end{array}$ & GC-MS/MS & $\begin{array}{l}0.8-66 \mathrm{ng} \mathrm{I}^{-1} \\
31-3189 \mathrm{ng} \mathrm{I}^{-1} \\
16-62600 \mathrm{ng} \mathrm{I}^{-1} \\
\text { / } 14\end{array}$ & $\begin{array}{l}\text { Tripha- } \\
\text { sic }\end{array}$ & $\begin{array}{l}\text { PRC } \\
\text { Laboratory calibra- } \\
\text { tion }\end{array}$ & $\begin{array}{l}\text { (Vallejo et al. } \\
\text { 2013) }\end{array}$ \\
\hline Screening & $\begin{array}{l}\text { Anticonvulsives, anti- } \\
\text { depressants, NSAIDS, } \\
\text { antipyretics, stimulants }\end{array}$ & Surface waters & LC-MS/MS & $1-200 \mathrm{ng} \mathrm{I}^{-1} / 21$ & HLB & $\begin{array}{l}\text { Trace metals in } \\
\text { parallel }\end{array}$ & $\begin{array}{l}\text { (Vystavna et al. } \\
\text { 2013) }\end{array}$ \\
\hline $\begin{array}{l}\text { Screening } \\
\text { Calibration }\end{array}$ & $\begin{array}{l}\text { Antibiotics, chemotera- } \\
\text { peutics }\end{array}$ & WWTP & LC-MS/MS & 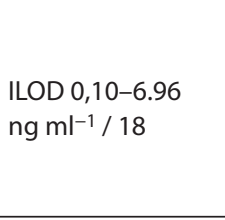 & o-DGT & $\begin{array}{l}\text { Novel passive } \\
\text { sampler device } \\
0.5 \mathrm{~mm} \text { XAD } 18 \\
\text { agarose binding gel, } \\
\text { a } 0.8 \mathrm{~mm} \text { standard } \\
\text { agarose diffusive gel }\end{array}$ & $\begin{array}{l}\text { (Chen et al. } \\
\text { 2013) }\end{array}$ \\
\hline $\begin{array}{l}\text { Screening } \\
\text { Bioassay }\end{array}$ & $\begin{array}{l}\text { Pharmaceuticals, illicit } \\
\text { drugs etc. }\end{array}$ & $\begin{array}{l}\text { Lake water and } \\
\text { sediment }\end{array}$ & LC-MS & $\begin{array}{l}1.6-5200 \mathrm{ng} \mathrm{I}^{-1} \\
\text { / ND }\end{array}$ & HLB & $\begin{array}{l}\text { YES } \\
\text { Distribution in } \\
\text { depth/sediment } \\
\text { SPMD in parallel }\end{array}$ & $\begin{array}{l}\text { (Alvarez et al. } \\
\text { 2012) }\end{array}$ \\
\hline
\end{tabular}




\begin{tabular}{|c|c|c|c|c|c|c|c|}
\hline Screening & Sulfonamides & $\begin{array}{l}\text { WWTP up- } \\
\text { stream } \\
\text { WWTP down- } \\
\text { stream }\end{array}$ & LC-MS/MS & $\begin{array}{l}<20-736 \mathrm{ng} \text { of } \\
\text { sulfamethoxaz- } \\
\text { ole equivalents } \\
\text { POCIS }^{-1} / 28\end{array}$ & $\begin{array}{l}\text { HLB } \\
\text { Tripha- } \\
\text { sic }\end{array}$ & $\begin{array}{l}\text { Combination with } \\
\text { ELISA }\end{array}$ & $\begin{array}{l}\text { (Cernoch et al. } \\
\text { 2012) }\end{array}$ \\
\hline $\begin{array}{l}\text { Screening } \\
\text { Calibration }\end{array}$ & $\beta$-blockers, hormones & $\begin{array}{l}\text { WWTP in/ } \\
\text { effluents, } \\
\text { WWTP up/ } \\
\text { downstream }\end{array}$ & LC-MS/MS & $\begin{array}{l}\text { > } 249 \text { ng sam- } \\
\text { pler }{ }^{-1} \text { atenolol, } \\
\text { sotalol } 66-113 \\
\text { ng.sampler-1 me- } \\
\text { toprolol, propran- } \\
\text { olol, bisoprolol } \\
\text { < } 4 \text { ng sampler } \\
\text { nadolol, timolol, } \\
\text { oxpenolol, betax- } \\
\text { olol } 355 \text { ng sam- } \\
\text { pler }^{-1} \text { estriol / } 24\end{array}$ & HLB & $\begin{array}{l}\text { TWA, PRC } \\
\text { In situ calibration }\end{array}$ & $\begin{array}{l}\text { (Jacquet et al. } \\
\text { 2012) }\end{array}$ \\
\hline $\begin{array}{l}\text { Screening } \\
\text { Bioassay }\end{array}$ & $\begin{array}{l}\text { Antibiotics, chemother- } \\
\text { apeutics, antiprotozoal } \\
\text { drugs, NSAIDs, anticon- } \\
\text { vulsives }\end{array}$ & $\begin{array}{l}\text { WWTP up/ } \\
\text { downstream }\end{array}$ & LC-MS/MS & 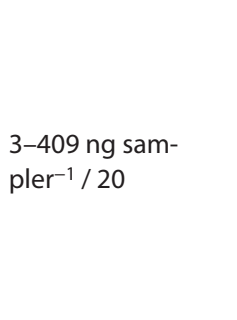 & $\begin{array}{l}\text { HLB } \\
\text { Tripha- } \\
\text { sic }\end{array}$ & $\begin{array}{l}\text { Estrogen activities } \\
\text { Non-specific } \\
\text { cytotoxicity, en- } \\
\text { docrine-disruptive } \\
\text { (ED) potential and } \\
\text { dioxin-like toxicity } \\
\text { MVLN, H4IIE-luc } \\
\text { recombinant } \\
\text { S. cerevisiae }\end{array}$ & $\begin{array}{l}\text { (Jarosova et al. } \\
\text { 2012) }\end{array}$ \\
\hline $\begin{array}{l}\text { Screening } \\
\text { Calibration } \\
\text { Bioassay }\end{array}$ & $\begin{array}{l}\text { Antibiotics, ampheta- } \\
\text { mines, opioids, marker } \\
\text { of untreated human } \\
\text { waste }\end{array}$ & Surface waters & LC-MS/MS & $0.5-71 \mathrm{ng} \mathrm{l}^{-1} / 30$ & ND & $\begin{array}{l}\text { YES } \\
\text { Grab sampling in } \\
\text { parallel }\end{array}$ & $\begin{array}{l}\text { (Jones-Lepp et } \\
\text { al. 2012) }\end{array}$ \\
\hline Screening & $\begin{array}{l}\text { Alkylphenols oes- } \\
\text { trogen hormones, } \\
\text { antidepressants, NSAIDs, } \\
\beta \text {-blockers, bronchodi- } \\
\text { lators, hypolipidemics, } \\
\text { stimulants }\end{array}$ & $\begin{array}{l}\text { WWTP effluent } \\
\text { WWTP up/ } \\
\text { downstream }\end{array}$ & LC-MS/MS & $\begin{array}{l}\text { ND-1000 ng sam- } \\
\text { pler }-1 / 28^{-128}\end{array}$ & HLB & $\begin{array}{l}\text { Sampling rates, PRC } \\
\text { studies } \\
\text { In situ and laborato- } \\
\text { ry calibration }\end{array}$ & $\begin{array}{l}\text { (Miege et al. } \\
\text { 2012) }\end{array}$ \\
\hline Screening & $\begin{array}{l}\text { Antidepressant, NSAIDs, } \\
\text { stimulants, anticonvul- } \\
\text { sives, bronchodilators, } \\
\text { hypolipidemics, alkyl- } \\
\text { phenols }\end{array}$ & Coastal waters & LC-MS/MS & up to $41 \mathrm{ng} \mathrm{l}^{-1} / 28$ & HLB & $\begin{array}{l}\text { Mediterranean } \\
\text { water }\end{array}$ & $\begin{array}{l}\text { (Munaron et al. } \\
\text { 2012) }\end{array}$ \\
\hline Screening & $\begin{array}{l}\text { Psychiatric drugs, anal- } \\
\text { gesics, broncholidators, } \\
\text { NSAIDs, hypolipidemics, } \\
\text { stimulants }\end{array}$ & $\begin{array}{l}\text { WWTP up/ } \\
\text { downstream }\end{array}$ & LC-MS/MS & $\begin{array}{l}\text { Approx. up to } 275 \\
\mathrm{ng} \cdot \mathrm{l}^{-1} / 21\end{array}$ & HLB & $\begin{array}{l}\text { Socioeconomic } \\
\text { study (Ukraine vs. } \\
\text { France) }\end{array}$ & $\begin{array}{l}\text { (Vystavna et al. } \\
2012 \text { ) }\end{array}$ \\
\hline Calibration & $\begin{array}{l}\text { Analgesic, stimulants, } \\
\text { nicotine metabolite, } \\
\text { antihistamines steroid } \\
\text { hormones }\end{array}$ & WWTP effluent & LC-MS/MS & $\begin{array}{l}0.3 \pm 0.2-5.1 \pm 1.9 \\
\mathrm{ng} \mathrm{l}^{-1} / 28\end{array}$ & $\begin{array}{l}\text { HLB } \\
\text { Tripha- } \\
\text { sic }\end{array}$ & $\begin{array}{l}\text { Laboratory calibra- } \\
\text { tion } \\
36 \text { compounds } \\
\text { with no previously } \\
\text { reported } \mathrm{R}_{\mathrm{s}} \text { values } \\
\text { (+ agrichemicals) }\end{array}$ & $\begin{array}{l}\text { (Bartelt-Hunt } \\
\text { et al. 2011) }\end{array}$ \\
\hline $\begin{array}{l}\text { Screening } \\
\text { Calibration }\end{array}$ & $\begin{array}{l}\text { Cocaine and its metab- } \\
\text { olites, amphetamines, } \\
\text { morphine, nicotine } \\
\text { metabolite, } \beta \text {-blockers' } \\
\text { metabolite, antihista- } \\
\text { mines, anticonvulsives, } \\
\text { analgesics, antibiotics, } \\
\text { sulfonamides, anthel- } \\
\text { mintics }\end{array}$ & $\begin{array}{l}\text { Sewage treat- } \\
\text { ment works }\end{array}$ & $\begin{array}{l}\text { UHPLC-MS/ } \\
\text { MS }\end{array}$ & $5-6389 \mathrm{ng} \mathrm{l}^{-1} / 14$ & HLB & $\begin{array}{l}\text { Cetirizine back } \\
\text { calculation } \\
\text { In situ calibration }\end{array}$ & $\begin{array}{l}\text { (Harman et al. } \\
\text { 2011) }\end{array}$ \\
\hline $\begin{array}{l}\text { Screening } \\
\text { Bioassay }\end{array}$ & Steroid hormones & Surface water & LC-MS/MS & Not detected / 7 & HLB & $\begin{array}{l}\text { Endocrine effects } \\
\text { hepatic mRNA } \\
\text { expression estrogen } \\
\text { receptor a (ERa), } \\
\text { gonadal expres- } \\
\text { sion of P450 } \\
\text { aromatase A (caged } \\
\text { minnows) }\end{array}$ & $\begin{array}{l}\text { (Jeffries et al. } \\
\text { 2011) }\end{array}$ \\
\hline
\end{tabular}

European Journal of Environmental Sciences, Vol. 6, №. 1 


\begin{tabular}{|c|c|c|c|c|c|c|c|}
\hline Calibration & $\begin{array}{l}\text { NSAIDs, antihistamines, } \\
\text { steroid hormones, anti- } \\
\text { depressants, } \beta \text {-blockers, } \\
\text { anticonvulsives, chemo- } \\
\text { therapeutics }\end{array}$ & $\begin{array}{l}\text { Spiked deion- } \\
\text { ized lake and } \\
\text { dechlorinated } \\
\text { tap water }\end{array}$ & LC-MS/MS & ND & $\begin{array}{l}\text { HLB } \\
\text { MAX } \\
\text { MCX }\end{array}$ & $\begin{array}{l}\text { Dissolved organic } \\
\text { matter, pH effect } \\
\text { In house MAX, MCX } \\
\text { sorbent } \\
\text { Laboratory calibra- } \\
\text { tion }\end{array}$ & (Li et al. 2011) \\
\hline $\begin{array}{l}\text { Screening } \\
\text { Bioassay }\end{array}$ & $\begin{array}{l}\text { Antidepressant, steroid } \\
\text { hormones alkylphenols, } \\
\text { NSAIDs, stimulants, } \\
\text { anticonvulsives, hy- } \\
\text { polipidemics, analgesic, } \\
\text { bronchodilators }\end{array}$ & Surface water & LC-MS/MS & $\begin{array}{l}\text { 3-20 ng sam- } \\
\text { pler }^{-1}(\Sigma) / 30\end{array}$ & HLB & $\begin{array}{l}\text { Toxicity estrogenic, } \\
\text { anti-androgenic and } \\
\text { dioxin-like activities } \\
\text { MELN, MDA-kb2, } \\
\text { PLHC-1 }\end{array}$ & $\begin{array}{l}\text { (Tapie et al. } \\
\text { 2011) }\end{array}$ \\
\hline
\end{tabular}

ND - not defined

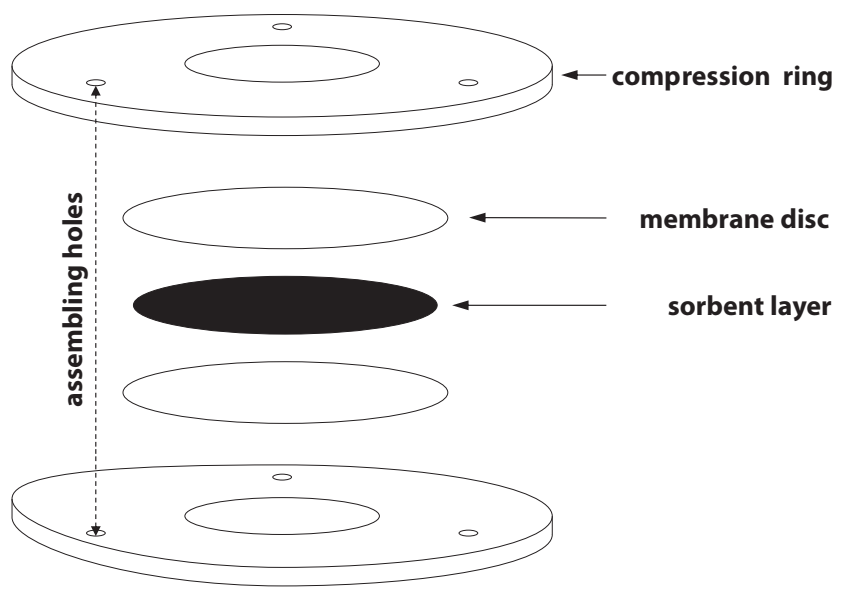

Fig. 4 Structure of the polar organic chemical integrative sampler.

configuration (POCISPest) contains a mixture of three sorbent materials and is aimed at sampling pesticides, natural and synthetic hormones, wastewater-related chemicals and other water-soluble organic chemicals. The sorbent mixture consists of a tri-phasic mixture of a hydroxylated polystyrene-divinylbenzene resin (Isolute ENV+) and a carbonaceous adsorbent (Ambersorb 1500) dispersed in a styrene divinylbenzene copolymer (S-X3 Bio Beads) (Alvarez et al. 2004). A "Pharmaceutical" configuration (POCISPharm) contains a single sorbent, usually Oasis HLB. Oasis HLB is designed for pharmaceutical sampling and is used in most studies of drug contaminants in aqueous environments since most of the classes of pharmaceuticals can be retained by a single sorbent. In comparison, there are limited advantages in using ion exchange sorbents in POCIS (Li et al. 2011). In some cases, both the receiving phases (HLB and triphasic) are deployed together to combine the advantages offered by the different mechanisms and so increase the chance of attracting more analytes (Cernoch et al. 2012; Jarosova et al. 2012; Liscio et al. 2014). Kaserzon et al. 2014 recently used POCIS containing a polymeric reverse phase, the Strata- $\mathrm{X}^{\mathrm{rm}}$, to study the kinetics of uptake of several ionizable and polar pesticides, pharmaceuticals and personal care products. Strata- $\mathrm{X}^{\mathrm{m}}$ sorbent performed similarly to Chemcatcher ${ }^{\text {me }}$ (SDB-RPS) and are both suitable monitoring tools.

The microporous membrane that covers the sorbent (Fig. 4) serves as a size-selective sieve (typically $0.1 \mu \mathrm{m}$ pores) that prevents biofouling, but might result in an altered sampling rate. Alvarez et al. (2004) evaluated several commercially available membranes for their use in a hydrophilic integrative sampler. Polyethersulfone (PES) exhibited the best combination: high analyte uptake rates, minimal superficial biofouling and membrane durability, necessary for long-term integrative sampling of polar organic chemicals. PES membrane is also used in both of the commercially available POCIS configurations (Pharm and Pest) and is the most frequently used membrane. Indeed, membranes used in-house assembled samplers are well described in the literature. For instance, there are nylon membranes enabling the sampling of hydrophobic pollutants and with an improved rate of accumulation for other pollutants (Belles et al. 2014). An innovative approach was introduced by Chen et al. (Chen et al. 2012; Chen et al. 2013), which used a gel-based layer instead of a membrane. Such diffusive gradients in thinfilms (DGT) samplers were originally used for the measurement of inorganic compounds and were shown to be relatively independent of the rate of water flow (Zhang and Davison 1995). The new configuration initially enabled measurements of organic chemicals (o-DGT) in the laboratory (Chen et al. 2012). This method was later validated and used for the routine monitoring of various antibiotics in wastewater (Chen et al. 2013).

\section{Target Analytes and Sampling Sites}

The analytical processes used to assess hydrophobic compounds (Kow $>3$ ) in aquatic environments are quite well characterized and the use of passive samplers (e.g. SPMD, LDPE, silicone rubber) for routine environmental monitoring is already a well-accepted procedure. POCIS can be considered as a complementary method because of the good affinity of this method for compounds with a log Kow < 4 (Alvarez et al. 2004), which includes hydrophilic compounds such as pharmaceuticals, household and industrial products, hormones, herbicides and polar pesticides. 
Antibiotics and chemotherapeutics, antidepressants, anticonvulsants, steroid hormones, NSAIDs, antipyretics, antihistamines, $\beta$-blockers, illicit drugs, stimulants, insecticides, fragrances, surfactants, alkylphenol compounds are the most frequent classes of pharmaceuticals and personal care products discussed in the literature (Table 1). Indeed, the correlation between exposure to humans/domestic animals and the abundance of these compounds in the water environment is obvious. Screening studies usually cover a wide range of chemicals in order to monitor local contamination and/or its seasonal changes. Vystavna et al. $(2012 ; 2013)$ even compare the profiles obtained from two different regions with contrasting socio-economic conditions. In some cases, only a specific range of substances is included in the study. For instance, amphetamines were monitored in the sewage from an area suspected of illegal drug activities (Boles and Wells 2014). Another two studies are on the removal of illicit drugs during the treatment of sewage (Rodayan et al. 2014; Yargeau et al. 2014). Alvarez et al. (2013) and Jeffries et al. (2011) address the analysis of steroid hormones in their studies, whereas Cernoch et al. (2012) analyse only sulfonamides. A number of POCIS studies are also coupled to bioassays, evaluating endocrine-disruptive potential or dioxine-like activities (Jeffries et al. 2011; Tapie et al. 2011; Alvarez et al. 2012; Jarosova et al. 2012; Alvarez et al. 2013; Jalova et al. 2013; Creusot et al. 2014).

The inputs and outflows from wastewater treatment plants (WWTP) and the river upstream and downstream close to a WWTP, respectively, are common POCIS sampling sites. In some cases, specific regions are studied such as tropical waters (Bayen et al. 2014), Mediterranean coastal waters (Munaron et al. 2012) or estuarine waters (Bayen et al. 2014; Shi et al. 2014). POCIS are also deployed in agricultural watersheds (Jairnes-Correa et al. 2015), areas of pharmaceutical industrial activity or hospital effluents (Bailly et al. 2013), to investigate the degree of contamination by particular pollutants. Spiked water samples and laboratory tests are commonly used in studies on the kinetics of uptake of new micropollutants and evaluation of various physical effects on the sampler or the characterization of the innovative features of future POCIS (Li et al. 2011; Carlson et al. 2013; Morin et al. 2013; Belles et al. 2014; Kaserzon et al. 2014). These approaches often include a range of analytes with different physical-chemical properties (basic, acidic, phenolic and neutral) which provide a better insight into the new samplers' efficiency for monitoring various analytes.

\section{Calibration, Sampling Rates and Performance of a Reference Standard (PRC)}

It is difficult to obtain reliable information on the kinetics of uptake of polar compounds and the effects of environmental factors (e.g. temperature, turbulence and biofouling). Determination of sampling rate is not straightforward as it takes a lot of time to obtain reliable sampling rate values $\left(R_{S}\right)$. A standard calibration procedure is still lacking and different approaches often involve the use of original laboratory devices, which results in variability in the determined $R_{S}$ values. Even though it is also virtually impossible to consider all the factors operating in the field, laboratory calibration is still the most popular method for evaluating $R_{S}$ (Bartelt-Hunt et al. 2011; Li et al. 2011; Miege et al. 2012; Morin et al. 2013; Vallejo et al. 2013; Amdany et al. 2014a; Bayen et al. 2014; Belles et al. 2014; Di Carro et al. 2014; Kaserzon et al. 2014). In situ calibration, at specific sites is more reliable but so far has rarely been used (Harman et al. 2011; Jacquet et al. 2012; Miege et al. 2012; Shi et al. 2014) because of the complexity of the process. Shi et al. (2014) compare both field and laboratory sampling rates for assessing ATB and endocrine disruptive compounds in estuarine waters. In this case, the field sampling rates were significantly higher for most of the compounds studied. Miege et al. (2012) compare in situ, laboratory and existing literature values. The differences in $R_{S}$ values are ascribed to differences in the POCIS, temperature, turbulence or exposed surface area. These authors also report that the $R_{S}$ values (in situ calibration) tend to be lower in WWTP effluents, which are characterized by a higher conductivity, higher concentrations of particulate suspended matter and dissolved organic carbon. It should be noted, however, that some screening studies use previously reported $R_{S}$ values. To date, comprehensive reviews (Harman et al. 2012; Morin et al. 2012) of the $R_{S}$ values of polar compounds provide supporting material. The use of a performance reference standard (PRC), which can eliminate the influence of environmental factors (already established for hydrophobic samplers), is still limited for hydrophilic compounds. Since Mazzella et al. (2010) proposed deuterated deisopropyl-atrazine-d5 (DIA d5) as an appropriate PRC for polar herbicide sampling, other compounds have been tested (Jacquet et al. 2012; Miege et al. 2012; Vallejo et al. 2013; Belles et al. 2014). Several deuterated hormones and $\beta$-blockers are also suggested by Jacquet et al. 2012 . Of a variety of hormone and $\beta$-blockers tested by Miege et al. (2012) only deuterated atenonol appeared to be a good potential PRC. Two other deuterated compounds $\left(\left[{ }^{2} \mathrm{H}_{3}\right]-\mathrm{E} 2\right.$ and $\left.\left[{ }^{2} \mathrm{H}_{4}\right]-\mathrm{EQ}\right)$ were successfully used by Vallejo et al. (2013).

\section{Extraction and Analytical Techniques}

Extraction of the analyte from a receiving phase after in-field exposure is the next step in the sample processing and the method may vary depending on the sorbent used and the character of the analyte. Methanol $(\mathrm{MeOH})$ is usually used for the extraction when POCISPharm is used. Other extraction media, e.g. ACN (Amdany et al. 2014a), acetone (Tanwar et al. 2015), $\mathrm{MeOH} /$ water (Bailly et al. 2013; Creusot et al. 2014) or MeOH/dichloromethane (Vystavna et al. 2012; Creusot et al. 2013; 
Morin et al. 2013; Vystavna et al. 2013) are also suitable. Some authors adjust the $\mathrm{pH}$ in order to improve the extraction (Harman et al. 2011; Cernoch et al. 2012; Bayen et al. 2014; Shi et al. 2014). Fixed ratio of solvents (dichloromethane: $\mathrm{MeOH}$ : toluene, 8:1:1) is common for POCISPest sorbent extraction. Kaserzon et al. (2014) describe a procedure for extraction of Strata- $\mathrm{X}^{\text {tw }}$ sorbent using $\mathrm{MeOH}$, acetonitrile and acetone. POCIS containing $\mathrm{MCX}$ and MAX sorbents can be eluted using $\mathrm{MeOH}$ with an addition of $5 \%$ ammonium hydroxide and $2 \%$ formic acid, respectively (Li et al. 2011). A study dealing with the stability of pharmaceuticals and other polar organic compounds stored in POCIS and solid phase cartridges indicates they remain relatively stable and suitable for expost analysis for 20 months when stored at $-20^{\circ} \mathrm{C}$ (Carlson et al. 2013).

Liquid chromatography is the method of choice in the majority of the studies surveyed and gas chromatography in cases dealing with steroid hormones or alkylphenols because of their low solubility in water (Alvarez et al. 2013; Vallejo et al. 2013; Coes et al. 2014). Tandem mass spectrometry is almost always mandatory since the detection of trace amounts of pollutant is required. With these analytical approaches, concentrations of PPCPs in the field can be reliably determined within the range of ng $1^{-1}$ or ng sampler-1 (Table 1). LC with UV and fluorescence detection (FLD) was used by Amdany et al. (2014a), whereas Cernoch et al. successfully used a POCIS in combination with an immunochemical ELISA technique for a semi-quantitative screening of sulfonamides, which gave similar results to the LC-MS (Cernoch et al. 2012).

\section{Bioassays}

The extracts from POCIS can be further used to assess the biological effects of water contaminants. A yeast estrogen screen (YES) is commonly used to evaluate estrogen in the environment (Alvarez et al. 2012; JonesLepp et al. 2012; Alvarez et al. 2013), but is not suitable for accurately assessing estrogenic activity at lower concentrations (Alvarez et al. 2013). In comparison, E-Screen and T47D-KBluc bioassays are more sensitive. Multi-receptor in vitro bioassays are often used to determine overall non-specific cytotoxicity, endocrine-disruptive potential and dioxin-like toxicity (Jeffries et al. 2011; Tapie et al. 2011; Jarosova et al. 2012; Jalova et al. 2013; Creusot et al. 2014). Recombinant S. cerevisiae or MELN, MDA-kb2 and H4IIE-luc cell lines are the most commonly used. The extracts from SPMD (Alvarez et al. 2012; Jalova et al. 2013), LDPE, silicone strips (Liscio et al. 2014) or sediment samples subject to accelerated solvent extraction (Creusot et al. 2013) should also be measured in parallel in order to complete and compare the results of the biological testing of the POCIS extract, since they could contain different classes of compounds. For instance, the arylhydrocarbon-mediated potency in both SPMD and POCIS indicate that both hydrophobic and polar compounds contribute to the overall dioxin-like potential of samples (Jalova et al. 2013). These authors also report that the cytotoxicity of wastewater is not correlated with the estrogenic or androgenic potencies, which are primarily caused by steroidal estrogens. The cytotoxicity of the POCIS extracts in the yeast assay is associated with antibiotics and other pharmaceuticals (Jalova et al. 2013).

\section{Conclusions}

Despite its relatively long history, passive sampling is an innovative monitoring tool, particularly in the context of monitoring environmental pollutants. The main benefits of passive sampling are the simplification of the overall sampling procedure, reductions in cost and solvent use and miniaturization. In addition, there are many factors that need to be considered when passive sampling is used in monitoring programs. Probably, the most important of these are the environmental conditions (flow, biofouling, temperature), which can strongly affect the accumulation of the target analyte in the sampler. Thus, in order to accurately determine the sampling rates for a wide range of new contaminants further detailed research is needed.

Passive sampling of pharmaceuticals in aquatic environments is most often used for the determination of time-weighted average concentrations or environmental screening. Though the concentrations differ for individual pharmaceutical and personal care products, the concentration usually remains within the range of ng up to $\mu \mathrm{g}$ per litter.

It would be of great value to use POCIS samplers for assessing and managing groundwater pollution. Moreover, the improvement of existing procedures and the search for new methods of passive sampling could, in the future, lead to the inclusion of additional substances into the monitoring programs and a lowering of the limits of detection.

\section{Acknowledgements}

The research leading to these results has received funding from the Norwegian Financial Mechanism 2009-2014 and the Ministry of Education, Youth and Sports under Project Contract no. MSMT-23681/2015-1.

\section{REFERENCES}

Alvarez DA, Petty JD, Huckins JN, Jones-Lepp TL, Getting DT, Goddard JP, Manahan SE (2004) Development of a passive, in situ, integrative sampler for hydrophilic organic contaminants in aquatic environments. Environ Toxicol Chem 23: $1640-1648$. 
Alvarez DA, Cranor WL, Perkins SD, Clark RC, Smith SB (2008) Chemical and toxicologic assessment of organic contaminants in surface water using passive samplers. J Environ Qual 37: 1024-1033.

Alvarez DA, Rosen MR, Perkins SD, Cranor WL, Schroeder VL, Jones-Lepp TL (2012) Bottom sediment as a source of organic contaminants in Lake Mead, Nevada, USA. Chemosphere 88: 605-611.

Alvarez DA, Shappell NW, Billey LO, Bermudez DS, Wilson VS, Kolpin DW, Perkins SD, Evans N, Foreman WT, Gray JL et al. (2013) Bioassay of estrogenicity and chemical analyses of estrogens in streams across the United States associated with livestock operations. Water Res 47: 3347-3363.

Amdany R, Chimuka L, Cukrowska E (2014a) Determination of naproxen, ibuprofen and triclosan in wastewater using the polar organic chemical integrative sampler (POCIS): A laboratory calibration and field application. Water Sa 40: 407-414.

Amdany R, Chimuka L, Cukrowska E, Kukucka P, Kohoutek J, Tolgyessy P, Vrana B (2014b) Assessment of bioavailable fraction of POPS in surface water bodies in Johannesburg City, South Africa, using passive samplers: an initial assessment. Environ Monit Assess 186: 5639-5653.

Bartelt-Hunt SL, Snow DD, Damon-Powell T, Brown DL, Prasai G, Schwarz M, Kolok AS (2011) Quantitative evaluation of laboratory uptake rates for pesticides, pharmaceuticals, and steroid hormones using POCIS. Environ Toxicol Chem 30: 1412-1420.

Bayen S, Segovia E, Loh LL, Burger DF, Eikaas HS, Kelly BC (2014) Application of Polar Organic Chemical Integrative Sampler (POCIS) to monitor emerging contaminants in tropical waters. Sci Total Environ 482: 15-22.

Bailly E, Levi Y, Karolak S (2013) Calibration and field evaluation of polar organic chemical integrative sampler (POCIS) for monitoring pharmaceuticals in hospital wastewater. Environ Pollut 174: 100-105.

Belles A, Pardon P, Budzinski H (2014) Development of an adapted version of polar organic chemical integrative samplers (POCIS-Nylon). Anal Bioanal Chem 406: 1099-1110.

Benes P, Steinnes E (1974) In situ dialysis for determination of state of trace elements in natural waters. Water Res 8: 947-953.

Boles TH, Wells MJM (2014) Pilot survey of methamphetamine in sewers using a Polar Organic Chemical Integrative Sampler. Sci Total Environ 472: 9-12.

Booij K, Sleiderink HM, Smedes F (1998) Calibrating the uptake kinetics of semipermeable membrane devices using exposure standards. Environ Toxicol Chem 17: 1236-1245.

Carlson JC, Challis JK, Hanson ML, Wong CS (2013) Stability of pharmaceuticals and other polar organic compounds stored on polar organic chemical integrative samplers and solid-phase extraction cartridges. Environ Toxicol Chem 32: 337-344.

Cernoch I, Franek M, Diblikova I, Hilscherova K, Randak T, Ocelka T, Blaha L (2012) POCIS sampling in combination with ELISA: Screening of sulfonamide residues in surface and waste waters. J Environ Monitor 14: 250-257.

Chen CE, Zhang H, Jones KC (2012) A novel passive water sampler for in situ sampling of antibiotics. J Environ Monitor 14: 1523-1530.

Chen CE, Zhang H, Ying GG, Jones KC (2013) Evidence and Recommendations to Support the Use of a Novel Passive Water Sampler to Quantify Antibiotics in Wastewaters. Environ Sci Technol 47: 13587-13593.

Coes AL, Paretti NV, Foreman WT, Iverson JL, Alvarez DA (2014) Sampling trace organic compounds in water: A comparison of a continuous active sampler to continuous passive and discrete sampling methods. Sci Total Environ 473: 731-741.
Creusot N, Ait-Aissa S, Tapie N, Pardon P, Brion F, Sanchez W, Thybaud E, Porcher JM, Budzinski H (2014) Identification of Synthetic Steroids in River Water Downstream from Pharmaceutical Manufacture Discharges Based on a Bioanalytical Approach and Passive Sampling. Environ Sci Technol 48: 3649-3657.

Creusot N, Tapie N, Piccini B, Balaguer P, Porcher JM, Budzinski H, Ait-Aissa S (2013) Distribution of steroid- and dioxin-like activities between sediments, POCIS and SPMD in a French river subject to mixed pressures. Environ Sci Pollut R 20: 2784-2794.

Di Carro M, Bono L, Magi E (2014) A simple recirculating flow system for the calibration of polar organic chemical integrative samplers (POCIS). Effect of flow rate on different water pollutants. Talanta 120: 30-33.

Endo S, Pfennigsdorff A, Goss KU (2012) Salting-Out Effect in Aqueous $\mathrm{NaCl}$ Solutions: Trends with Size and Polarity of Solute Molecules. Environ Sci Technol 46: 1496-1503.

Fedorova G, Randak T, Golovko O, Kodes V, Grabicova K, Grabic $\mathrm{R}$ (2014) A passive sampling method for detecting analgesics, psycholeptics, antidepressants and illicit drugs in aquatic environments in the Czech Republic. Sci Total Environ 487: 681-687.

Fowler WK (1982) Fundamentals of passive vapor sampling. Am Lab 14: 80-87.

Harman C, Allan IJ, Vermeirssen ELM (2012) Calibration and use of the polar organic chemical integrative sampler - a critical review. Environ Toxicol Chem 31: 2724-2738.

Harman C, Reid M, Thomas KV (2011) In Situ Calibration of a Passive Sampling Device for Selected Illicit Drugs and Their Metabolites in Wastewater, And Subsequent Year-Long Assessment of Community Drug Usage. Environ Sci Technol 45: 5676-5682.

Hoque ME, Cloutier F, Arcieri C, McInnes M, Sultana T, Murray C, Vanrolleghem PA, Metcalfe CD (2014) Removal of selected pharmaceuticals, personal care products and artificial sweetener in an aerated sewage lagoon. Sci Total Environ 487: 801-812.

Huckins JN, Petty JD, Booij K (2006) Monitors of Organic Chemicals in the Environment: Semipermeable Membrane Devices. Springer, New York.

Huckins JN, Tubergen MW, Manuweera G (1990) Semipermeable membrane devices containing model lipid - a new approach to monitoring the bioavailability of lipophilic contaminants and estimating their bioconcentration potential. Chemosphere 20: 533-552.

Jacquet R, Miege C, Bados P, Schiavone S, Coquery M (2012) Evaluating the polar organic chemical integrative sampler for the monitoring of beta-blockers and hormones in wastewater treatment plant effluents and receiving surface waters. Environ Toxicol Chem 31: 279-288.

Jaimes-Correa JC, Snow DD, Bartelt-Hunt SL (2015) Seasonal occurrence of antibiotics and a beta agonist in an agriculturally-intensive watershed. Environ Pollut 205: 87-96.

Jalova V, Jarosova B, Blaha L, Giesy JP, Ocelka T, Grabic R, Jurcikova J, Vrana B, Hilscherova K (2013) Estrogen-, androgen- and aryl hydrocarbon receptor-mediated activities in passive and composite samples from municipal waste and surface waters. Environ Int 59: 372-383.

Jarosova B, Blaha L, Vrana B, Randak T, Grabic R, Giesy JP, Hilscherova K (2012) Changes in concentrations of hydrophilic organic contaminants and of endocrine-disrupting potential downstream of small communities located adjacent to headwaters. Environ Int 45: 22-31. 
Jeffries MKS, Abbott KI, Cowman T, Kolok AS (2011) Occurrence and endocrine effects of agrichemicals in a small Nebraska, USA, watershed. Environ Toxicol Chem 30: 2253-2260.

Jones-Lepp TL, Sanchez C, Alvarez DA, Wilson DC, Taniguchi-Fu RL (2012) Point sources of emerging contaminants along the Colorado River Basin: Source water for the arid Southwestern United States. Sci Tot Environ 430: 237-245.

Kaserzon SL, Hawker DW, Kennedy K, Bartkow M, Carter S, Booij K, Mueller JF (2014) Characterisation and comparison of the uptake of ionizable and polar pesticides, pharmaceuticals and personal care products by POCIS and Chemcatchers. Environ Sci -Proc Imp 16: 2517-2526.

Kot-Wasik A, Zabiegala B, Urbanowicz M, Dominiak E, Wasik A, Namiesnik J (2007) Advances in passive sampling in environmental studies. Anal Chim Acta 602: 141-163.

Kylin H, Grimvall E, Ostman C (1994) Environmental monitoring of polychlorinated-biphenyls using pine needles as passive samplers. Environ Sci Technol 28: 1320-1324.

Li HX, Helm PA, Paterson G, Metcalfe CD (2011) The effects of dissolved organic matter and $\mathrm{pH}$ on sampling rates for polar organic chemical integrative samplers (POCIS). Chemosphere 83: 271-280.

Liscio C, Abdul-Sada A, Al-Salhi R, Ramsey MH, Hill EM (2014) Methodology for profiling anti-androgen mixtures in river water using multiple passive samplers and bioassay-directed analyses. Water Res 57: 258-269.

Lydy MJ, Landrum PF, Oen AMP, Allinson M, Smedes F, Harwood AD, Li HZ, Maruya KA, Liu JF (2014) Passive sampling methods for contaminated sediments: State of the science for organic contaminants. Integr Environ Asses 10: 167-178.

Mazzella N, Lissalde S, Moreira S, Delmas F, Mazellier P, Huckins JN (2010) Evaluation of the use of performance reference compounds in an Oasis-HLB adsorbent-based passive sampler for improving water concentration estimates of polar herbicides in freshwater. Environ Sci Technol 44: 1713-1719.

Metcalfe C, Hoque ME, Sultana T, Murray C, Helm P, Kleywegt $S$ (2014) Monitoring for contaminants of emerging concern in drinking water using POCIS passive samplers. Environ SciProc Imp 16: 473-481.

Miege C, Budzinski H, Jacquet R, Soulier C, Pelte T, Coquery M (2012) Polar organic chemical integrative sampler (POCIS): application for monitoring organic micropollutants in wastewater effluent and surface water. J Environ Monitor 14: 626-635.

Mills GA, Gravell A, Vrana B, Harman C, Budzinski H, Mazzella N, Ocelka T (2014) Measurement of environmental pollutants using passive sampling devices - an updated commentary on the current state of the art. Environ Sci - Proc Imp 16: 369-373.

Mills GA, Vrana B, Allan I, Alvarez DA, Huckins JN, Greenwood $\mathrm{R}$ (2007) Trends in monitoring pharmaceuticals and personal-care products in the aquatic environment by use of passive sampling devices. Anal Bioanal Chem 387: 1153-1157.

Morin N, Miege C, Randon J, Coquery M (2012) Chemical calibration, performance, validation and applications of the polar organic chemical integrative sampler (POCIS) in aquatic environments. Trac-Trend Anal Chem 36: 144-175.

Morin N, Camilleri J, Cren-Olive C, Coquery M, Miege C (2013) Determination of uptake kinetics and sampling rates for 56 organic micropollutants using "pharmaceutical" POCIS. Talanta 109: 61-73.

Munaron D, Tapie N, Budzinski H, Andral B, Gonzalez JL (2012) Pharmaceuticals, alkylphenols and pesticides in Mediterranean coastal waters: Results from a pilot survey using passive samplers. Estuar Coast Shelf S 114: 82-92.
Ouyang G, Zhao W, Alaee M, Pawliszyn J (2007) Time-weighted average water sampling with a diffusion-based solid-phase microextraction device. J Chromatogr A 1138: 42-46.

Palmes ED, Gunnison AF (1973) Personal monitoring device for gaseous contaminants. Am Ind Hyg Assoc J 34: 78-81.

Rodayan A, Majewsky M, Yargeau V (2014) Impact of approach used to determine removal levels of drugs of abuse during wastewater treatment. Sci Total Environ 487: 731-739.

Rose VE, Perkins JL (1982) Passive dosimetry - state of the art review. Am Ind Hyg Assoc J 43: 605-621.

Schilderman P, Moonen EJC, Maas LM, Welle I, Kleinjans JCS (1999) Use of crayfish in biomonitoring studies of environmental pollution of the river Meuse. Ecotox Environ Safe 44: 241-252.

Seethapathy S, Gorecki T, Li X (2008) Passive sampling in environmental analysis. J Chromatogr A 1184: 234-253.

Shi X, Zhou JL, Zhao H, Hou L, Yang Y (2014) Application of passive sampling in assessing the occurrence and risk of antibiotics and endocrine disrupting chemicals in the Yangtze Estuary, China. Chemosphere 111: 344-351.

Tanwar S, Di Carro M, Magi E (2015) Innovative sampling and extraction methods for the determination of nonsteroidal anti-inflammatory drugs in water. J Pharmaceut Biomed 106: 100-106.

Tapie N, Devier MH, Soulier C, Creusot N, Le Menach K, Ait-Aissa S, Vrana B, Budzinski H (2011) Passive samplers for chemical substance monitoring and associated toxicity assessment in water. Water Sci Technol 63: 2418-2426.

Turja R, Lehtonen KK, Meierjohann A, Brozinski JM, Vahtera E, Soirinsuo A, Sokolov A, Snoeijs P, Budzinski H, Devier MH, Peluhet L, Paakkonen JP, Viitasalo M, Kronberg L (2015) The mussel caging approach in assessing biological effects of wastewater treatment plant discharges in the Gulf of Finland (Baltic Sea). Mar Pollut Bull 97: 135-149.

Vallejo A, Prieto A, Moeder M, Usobiaga A, Zuloaga O, Etxebarria N, Paschke A (2013) Calibration and field test of the Polar Organic Chemical Integrative Samplers for the determination of 15 endocrine disrupting compounds in wastewater and river water with special focus on performance reference compounds (PRC). Water Res 47: 2851-2862.

van der Oost R, Beyer J, Vermeulen NPE (2003) Fish bioaccumulation and biomarkers in environmental risk assessment: a review. Environ Toxicol Pharm 13: 57-149.

Verreydt G, Bronders J, Van Keer I, Diels L, Vanderauwera P (2010) Passive Samplers for Monitoring VOCs in Groundwater and the Prospects Related to Mass Flux Measurements. Ground Water Monit R 30: 114-126.

Vrana B, Mills GA, Allan IJ, Dominiak E, Svensson K, Knutsson J, Morrison G, Greenwood R (2005) Passive sampling techniques for monitoring pollutants in water. Trac-Trend Anal Chem 24: $845-868$.

Vrana B, Mills GA, Dominiak E, Greenwood R (2006) Calibration of the Chemcatcher passive sampler for the monitoring of priority organic pollutants in water. Environ Pollut 142: 333-343.

Vystavna Y, Huneau F, Grynenko V, Vergeles Y, Celle-Jeanton H, Tapie N, Budzinski H, Le Coustumer P (2012) Pharmaceuticals in Rivers of Two Regions with Contrasted Socio-Economic Conditions: Occurrence, Accumulation, and Comparison for Ukraine and France. Water Air Soil Poll 223: 2111-2124.

Vystavna Y, Le Coustumer P, Huneau F (2013) Monitoring of trace metals and pharmaceuticals as anthropogenic and socio-economic indicators of urban and industrial impact on surface waters. Environ Monit Assess 185: 3581-3601. 
Yargeau V, Taylor B, Li HX, Rodayan A, Metcalfe CD (2014) Analysis of drugs of abuse in wastewater from two Canadian cities. Sci Total Environ 487: 722-730.

Yargicoglu EN, Reddy KR (2015) Review of biological diagnostic tools and their applications in geoenvironmental engineering. Rev Environ Sci Bio 14: 161-194.

Zabiegala B, Kot-Wasik A, Urbanowicz M, Namiesnik J (2010) Passive sampling as a tool for obtaining reliable analytical information in environmental quality monitoring. Anal Bioanal Chem 396: 273-296.
Zenobio JE, Sanchez BC, Leet JK, Archuleta LC, Sepulveda MS (2015) Presence and effects of pharmaceutical and personal care products on the Baca National Wildlife Refuge, Colorado. Chemosphere 120: 750-755.

Zhang H, Davison W (1995) Performance characteristics of diffusion gradients in thin films for the in situ measurement of trace metals in aqueous solution. Anal Chem 67: 3391-3400. 